\title{
Genome-wide analysis of maltose utilization and regulation in aspergilli
}

\author{
Wanwipa Vongsangnak, ${ }^{1} \dagger$ Margarita Salazar, ${ }^{1} \dagger$ Kim Hansen ${ }^{2}$ \\ and Jens Nielsen ${ }^{1}$ \\ ${ }^{1}$ Department of Chemical and Biological Engineering, Chalmers University of Technology, SE-412 \\ 96 Gothenburg, Sweden \\ ${ }^{2}$ Novozymes A/S, DK-2880 Bagsvaerd, Denmark
}

Correspondence

Jens Nielsen

nielsenj@chalmers.se

Received 22 May 2009

Revised 4 August 2009

Accepted 17 August 2009

\begin{abstract}
Maltose utilization and regulation in aspergilli is of great importance for cellular physiology and industrial fermentation processes. In Aspergillus oryzae, maltose utilization requires a functional $M A L$ locus, composed of three genes: MALR encoding a regulatory protein, MALT encoding maltose permease and MALS encoding maltase. Through a comparative genome and transcriptome analysis we show that the MAL regulon system is active in $A$. oryzae while it is not present in Aspergillus niger. In order to utilize maltose, $A$. niger requires a different regulatory system that involves the AmyR regulator for glucoamylase ( $\mathrm{gla} A$ ) induction. Analysis of reporter metabolites and subnetworks illustrates the major route of maltose transport and metabolism in $A$. oryzae. This demonstrates that overall metabolic responses of $A$. oryzae occur in terms of genes, enzymes and metabolites when the carbon source is altered. Although the knowledge of maltose transport and metabolism is far from being complete in Aspergillus spp., our study not only helps to understand the sugar preference in industrial fermentation processes, but also indicates how maltose affects gene expression and overall metabolism.
\end{abstract}

\section{INTRODUCTION}

Aspergillus represents a large genus of filamentous fungi, and several species have a long history of application as cell factories in the fermentation and food industries. In China and Japan, Aspergillus oryzae is used for the production of alcoholic beverages, soy bean paste, soy sauce and rice vinegar as well as for the production of various hydrolytic enzymes, such as $\alpha$-amylase (EC 3.2.1.1) (Baker \& Bennett, 2008). Aspergillus niger is largely exploited for the production of extracellular enzymes, such as glucoamylase (EC 3.2.1.3) (Baker \& Bennett, 2008; Magnuson \& Lasure, 2004). Maltose utilization and regulation in aspergilli is of great importance for academic and industrial research. Maltose is one of the most effective inducers for enzyme production in aspergilli such as $\alpha$-amylase production by A. oryzae (Carlsen et al., 1996; Carlsen \& Nielsen, 2001), but also for glucoamylase production measured by enzymic activity in A. niger and Aspergillus nidulans (Kato et al.,

†These authors contributed equally to this work.

Abbreviation: TA, transcriptional analysis.

Normalized gene expression data have been deposited at the GEO database (http://www.ncbi.nlm.nih.gov/geo/), with accession numbers GPL5975 (platform), GSM348998-GSM349003 (samples of $A$. oryzae), GSM349007-GSM349012 (samples of $A$. niger), and GSE13868 (series).

Supplementary material is available with the online version of this paper.
2002; Barton et al., 1972). However, compared to the yeast Saccharomyces cerevisiae little is known about maltose utilization, transport and regulation at the molecular level in aspergilli. Regulation of maltose transport and metabolism by yeast is well known (Klein et al., 1996; Novak et al., 2004). Maltose utilization in S. cerevisiae is under control of three general regulatory mechanisms: induction, glucose repression and glucose inactivation (Novak et al., 2004). The presence of maltose in the environment is necessary for the induction of synthesis of maltase and maltose transporter. The metabolism and regulation of maltose requires the presence of $M A L$ loci, of which several have been identified in different strains of $S$. cerevisiae, but the MAL6 locus is the best studied (Klein et al., 1996). The MAL6 locus is composed of a cluster of three genes: MAL61 (MALT) encoding maltose permease, MAL62 (MALS) encoding maltase (EC 3.2.1.20) and MAL63 (MALR), encoding a transcriptional activator specifically activating expression of the MALT and MALS genes (Needleman et al., 1984). Expression of both MALT and MALS is carbon catabolite repressed by glucose through the transcription factor Mig1 and coordinately induced by maltose (Klein et al., 1996).

In recent years, availability of genome sequences of filamentous fungi such as Aspergillus species has allowed for the study of metabolism in greater detail, e.g. maltose metabolism and transport, as well as filling existing gaps at 
the molecular level. Since the release of ten different Aspergillus species genome sequences (Baker, 2006; Fedorova et al., 2008; Galagan et al., 2005; Machida et al., 2005; Nierman et al., 2005; Payne et al., 2006; Pel et al., 2007; Yu et al., 2005), several transcriptome analyses have been conducted with some of these sequences (Breakspear \& Momany, 2007), and recently we developed an Affymetrix GeneChip that allows for transcriptome analysis of any of the three aspergilli $A$. oryzae, A. niger and $A$. nidulans (Andersen et al., 2008). In this study, we aim to identify the $M A L$ gene cluster in different sequenced Aspergillus genomes using the gene structure of the MAL6 locus of $S$. cerevisiae as a model. We further validate the presence or absence of the MAL gene cluster in A. oryzae and $A$. niger by using our custom-designed Affymetrix GeneChip for transcriptome analysis (Andersen et al., 2008). To do this, we performed batch cultivations of two Aspergillus species (A. oryzae and A. niger) on glucose and maltose as carbon source, and we then performed comparative transcriptome analysis to examine expression of putative MAL gene clusters in these two species. Furthermore, our study allowed for mapping the transcriptional response onto the metabolic network of $A$. oryzae when adjusting metabolism to a change of carbon source, from glucose to maltose.

\section{METHODS}

Strains. The strains used were A. oryzae wild-type strain A1560 (an ancestor of strains used for commercial $\alpha$-amylase production) and $A$. niger wild-type strain BO1 (an ancestor of strains used for commercial glucoamylase production), both obtained from Novozymes (Carlsen \& Nielsen, 2001; Pedersen et al., 2000). A. oryzae stock culture was maintained on Cove-N-Gly agar at $4{ }^{\circ} \mathrm{C}$ and $A$. niger stock culture was maintained as frozen spore suspensions at $-80{ }^{\circ} \mathrm{C}$ in $20 \%(\mathrm{v} / \mathrm{v})$ glycerol.

Medium and inoculum. The details of spore propagation medium, pre-culture medium, and batch cultivation medium for $A$. oryzae or A. niger are described in Supplementary file 1, available with the online version of this paper. For the initial fermentation process, $A$. oryzae A1560 fermenters were inoculated with $\sim 60 \mathrm{~g}$ broth of $A$. oryzae A1560 cultured at $30{ }^{\circ} \mathrm{C}$ for $24 \mathrm{~h}$ on pre-culture liquid medium in shake flasks at 250 r.p.m. The pre-culture was inoculated with $5 \mathrm{ml}$ spore solution harvested from mycelium grown on spore propagation medium agar at $34{ }^{\circ} \mathrm{C}$ for $3-4$ days. Spores were harvested with $0.1 \%(\mathrm{v} / \mathrm{v})$ Tween 80 . The A. niger fermenters were inoculated with spores $\left(5.7 \times 10^{9}\right.$ spores $\left.1^{-1}\right)$ previously propagated on spore propagation medium for 8 days at $30{ }^{\circ} \mathrm{C}$. Spores were harvested by adding $0.01 \%(\mathrm{v} / \mathrm{v})$ Tween 80 .

Batch cultivations. A. oryzae batch cultivations were done in 21 bioreactors with a working volume of 1.21 . The stirrer speed was kept at 800 r.p.m. during the first $4 \mathrm{~h}$ and then increased to 1100 r.p.m. The $\mathrm{pH}$ was controlled at 6 by addition of $10 \%(\mathrm{v} / \mathrm{v}) \mathrm{H}_{3} \mathrm{PO}_{4}$ or $10 \%$ (v/v) $\mathrm{NH}_{3}$ solution, and the temperature was maintained at $34{ }^{\circ} \mathrm{C}$. The aeration flow rate was set at $1.2 \mathrm{vvm}$ (volume of gas per volume of liquid per minute). Dissolved oxygen tension was initially calibrated at $100 \%$. The concentrations of oxygen $\left(\mathrm{O}_{2}\right)$ and carbon dioxide $\left(\mathrm{CO}_{2}\right)$ in the exhaust gas were monitored with a gas analyser (Magnos 4G for $\mathrm{O}_{2}$, Uras $3 \mathrm{G}$ for $\mathrm{CO}_{2}$; Hartmann \& Braun).
A. niger batch cultivations were carried out in 4.51 bioreactors with a working volume of 41 . The bioreactors were equipped with two Rushton four-blade disc turbines, $\mathrm{pH}$ and temperature control and no baffles. Inlet air was controlled with a mass flow meter. The concentrations of $\mathrm{O}_{2}$ and $\mathrm{CO}_{2}$ in the exhaust gas were monitored with a gas analyser (1311 Fast response Triple gas, Innova, combined with multiplexer controller for Gas Analysis MUX100, B. Braun Biotech International). The temperature was maintained at $30{ }^{\circ} \mathrm{C}$ and the $\mathrm{pH}$ was controlled by automatic addition of $2 \mathrm{M} \mathrm{NaOH}$. The $\mathrm{pH}$ was initially set to 3.0 to prevent spore aggregation. Once the spores started to germinate, the $\mathrm{pH}$ was increased to 4.5 and kept constant throughout the cultivation. The stirring speed was initially set to 200 r.p.m. and the aeration rate was set to 0.05 vvm. After germination, the stirring speed was increased to 600 r.p.m. and aeration rate was raised to $0.89 \mathrm{vvm}$ and kept steady throughout all the rest of the fermentation.

Sampling. Cell dry weight was determined by filtration. A known volume of cell culture was filtered, and then dried on the filter at $100{ }^{\circ} \mathrm{C}$ for $24 \mathrm{~h}$ and cooled in a desiccator. The filter with dried cell mass was weighed afterwards. The culture supernatant was obtained after centrifugation of original samples and subsequently frozen at $-20{ }^{\circ} \mathrm{C}$ for measurements of sugars and extracellular metabolites. For gene expression analysis, mycelium was harvested at the early to midexponential phase and then cultures were filtered through sterile filtration Miracloth (Calbiochem). At this point, the mycelium was washed with distilled water or $0.9 \%(\mathrm{w} / \mathrm{v}) \mathrm{NaCl}$ solution. The mycelium was quickly dried by squeezing and subsequently frozen in liquid nitrogen. Samples were stored at $-80{ }^{\circ} \mathrm{C}$ until RNA extraction.

Measurements of sugars and extracellular metabolites. The concentrations of sugars and extracellular metabolites were measured by HPLC analysis on an Aminex HPX-87H ion-exclusion column (Bio-Rad) with previous filtration by $25 \mathrm{~mm}$ GD/X syringe filter, $0.45 \mu \mathrm{m}$ pore size (Whatman). The column was eluted at $45{ }^{\circ} \mathrm{C}$ for $A$. oryzae or $60{ }^{\circ} \mathrm{C}$ for $A$. niger with $5 \mathrm{mM} \mathrm{H}_{2} \mathrm{SO}_{4}$ at a flow rate of $0.6 \mathrm{ml}$ $\mathrm{min}^{-1}$. Extracellular metabolites were detected with a refractive index detector and a UV detector.

Total RNA extraction. A. oryzae total RNA was extracted by using the Promega RNAgents Total RNA Isolation system, according to the protocol for purification of total RNA from fungi. For RNA extraction, $\sim 1 \mathrm{~g}$ frozen mycelium was ground to a fine powder under liquid nitrogen using a ceramic mortar and pestle. Total RNA of $A$. niger was isolated using the Qiagen RNeasy Mini kit, according to the protocol for isolation of total RNA from plants and fungi. For RNA isolation, $\sim 100 \mathrm{mg}$ frozen mycelium was placed in a $2 \mathrm{ml}$ tube, precooled in liquid nitrogen, containing three RNase-treated steel balls. The tubes were then shaken in a Mixer Mill, at $5{ }^{\circ} \mathrm{C}$ for $10 \mathrm{~min}$, until the mycelium was ground to powder, and thus ready for extraction of total RNA. For all samples, the quality of the total RNA extracted was determined by using a BioAnalyser (2100 BioAnalyser, Agilent Technologies) and the quantity determined by using a spectrophotometer (Amersham Pharmacia Biotech, GE Healthcare BioSciences). Total purified RNA was stored at $-80{ }^{\circ} \mathrm{C}$ until further microarray processing.

Microarray manufacturing and design. Affymetrix arrays were used for the analysis of the transcriptomes of $A$. oryzae and $A$. niger. The arrays were packaged in an Affymetrix GeneChip cartridge (49 format), and were processed with GeneChip reagents in the GeneChip Instrument System. The design and selection of probes for interrogating the ORFs within the genomes of $A$. oryzae and $A$. niger was performed by Andersen et al. (2008). The array contains only perfect match (PM) probes which correspond to 25-base oligonucleotides perfectly complementary to the transcript. Of the 13120 putative genes identified in the genome of A. oryzae (Machida et al., 
2005; Vongsangnak et al., 2008), 12039 probe sets were used for microarray analysis. Of the 11200 putative genes identified in the genome of A. niger, 11122 probe sets were used for microarray analysis. Each of the probe sets was composed of 11 probes (whenever possible) of 25 oligomers (Andersen et al., 2008).

Biotin-labelled cRNA and microarray processing. Biotin-labelled cRNA was prepared from $\sim 5 \mu \mathrm{g}$ total RNA, according to the protocol described in the Affymetrix GeneChip Expression Analysis Technical Manual (Affymetrix \& GeneChip, 2007). The cRNA was cleaned before fragmentation by using the Qiagen RNeasy Mini kit (protocol for RNA Cleanup), in order to guarantee the quality of cRNA samples for further processing. Biotin-labelled cRNA was quantified in a spectrophotometer (Amersham Pharmacia Biotech, GE Healthcare Bio-Sciences). Then, $20 \mu \mathrm{g}$ cRNA was fragmented following the manufacturer's protocol and $\sim 15 \mu \mathrm{g}$ fragmented cRNA was hybridized to the Aspergillus Affymetrix chip (Andersen et al., 2008) according to the Affymetrix GeneChip Expression Analysis protocol (Affymetrix \& GeneChip, 2007). Arrays were washed and stained using a GeneChip Fluidics Station FS-400, and scanned on an Agilent GeneArray Scanner.

Microarray data acquisition and analysis. Affymetrix CEL-data files were preprocessed using bioconductor (Gentleman et al., 2004) and $\mathrm{R}$ package version 2.5.1 ( $\mathrm{R}$ Development Core Team). Normalization was performed by using the qspline algorithm (Workman et al., 2002). The normalized gene expression dataset is presented in Supplementary file 2. The probe intensities were corrected for background by using the robust multiarray average method (Irizarry et al., 2003) by using all the probes. Gene expression values were calculated from the probes associated with each gene with the median polish summary method (Irizarry et al., 2003). All statistical preprocessing methods were invoked through the affy package (Gautier et al., 2004) and R scripts (Dudoit et al., 2003). Statistical analysis was applied to determine significantly different gene expressions. The limma package (Smyth et al., 2005) was used to perform moderated Student's $t$-tests for pair-wise carbon source comparisons. Empirical Bayesian statistics were used to moderate the standard errors within each gene and Benjamini-Hochberg's method to adjust for multi-testing (Benjamini \& Hochberg, 1995). A cut-off value of adjusted $P<0.05$ was set to assess statistical significance.

Identification of the MAL gene cluster based on protein sequence analysis. A cluster of three genes from the $S$. cerevisiae MAL6 locus was used as a scaffold model to identify the MAL gene cluster in 10 Aspergillus genome sequences. The three S. cerevisiae genes used were MAL61, MAL62 and MAL63, amino acid sequences of which were extracted from the GenBank database (http:// www.ncbi.nlm.nih.gov/) with accession numbers P15685.1, P07265.1 and P10508.1. The complete set of three amino acid sequences of $S$. cerevisiae was further used as query and searched against the amino acid sequences of 10 different sequenced Aspergillus genomes by using BLASTP (Altschul et al., 1990). The sequenced species included were: A. oryzae RIB40 (Machida et al., 2005), A. niger CBS 513.88 (Pel et al., 2007), A. niger ATCC 1015 (version 3.0) (http://genome.jgi-psf.org/Aspni5/Aspni5.home.html), A. nidulans FGSC A4 (version 4) (Galagan et al., 2005; Wortman et al., 2009), Aspergillus fumigatus Af293 (Nierman et al., 2005), Aspergillus fumigatus A1163 (Fedorova et al., 2008), Aspergillus flavus NRRL 3357 (Payne et al., 2006), Aspergillus terreus NIH2624 (www.broadinstitute. org/annotation/genome/aspergillus_group/MultiDownloads.html), Aspergillus clavatus NRRL 1 (Fedorova et al., 2008) and Aspergillus fischeri NRRL 181 (Fedorova et al., 2008). An estimated expectation value cut-off of less than $1 \mathrm{E}-100$, more than $40 \%$ identity, and more than $500 \mathrm{bp}$ of alignment length was set to assess statistical significance for identification of any orthologues. For identification of the $M A L$ gene cluster based on synteny analysis, the expectation value cut-off of less than 1E-05, more than $20 \%$ identity, and more than 200 bp of alignment length was set to assess statistical significance.

Reporter metabolites and subnetwork analysis. The reporter metabolites and highly correlated metabolic subnetwork algorithm was applied as described by Patil \& Nielsen (2005). The analysis was run for A. oryzae, for the glucose versus maltose carbon source pairwise comparison. For this purpose, information on the topology of the reconstructed metabolic network of A. oryzae (Vongsangnak et al., 2008) was used in combination with the adjusted $P$-values obtained from the Student's $t$-test analysis.

\section{RESULTS}

\section{Comparative analysis of the MAL gene cluster in S. cerevisiae and Aspergillus species}

First we searched for the presence of the MAL gene cluster in 10 different sequenced Aspergillus genomes. For this purpose, the gene structure of the MAL6 locus of $S$. cerevisiae was used as a model and BLASTP was applied (see Methods). The results showed that six different Aspergillus strains (i.e. A. oryzae, two strains of A. fumigatus, A. flavus, A. clavatus, and A. fischeri) contain at least one $M A L$ gene cluster as illustrated in Fig. 1. Interestingly, A. oryzae and $A$. flavus contain at least two MAL gene clusters. Phylogenetic analysis suggests that events of gene duplication and horizontal gene transfer may have occurred (see Supplementary file 3 ). In contrast, we could not find any $M A L$ cluster in A. nidulans, $A$. terreus and two strains of $A$. niger under the statistical constraints imposed. These results could suggest that these four Aspergillus strains most likely do not have the MAL regulon for maltose utilization. Notably, in all the sequenced Aspergillus genomes, we could identify multiple orthologue genes encoding maltase or $\alpha$-glucosidase enzymes and maltose transporters as shown in Fig. 1. Statistical values of orthologous genes are presented in Supplementary file 3.

To prove the presence or absence of the MAL gene cluster at the transcriptional level, we further evaluated our results obtained from comparative genomics through transcriptomics analysis. In the following, we show an example of using our previously designed Aspergillus GeneChip (Andersen et al., 2008) to validate the presence of the $M A L$ gene cluster in the A. oryzae genome and the absence of this gene cluster in the $A$. niger genome.

\section{Growth physiology}

To evaluate the physiology and validate the presence or absence of the MAL gene cluster as well as to analyse the regulatory response when adjusting metabolism to a change of carbon source, i.e. from glucose to maltose, we grew the two Aspergillus species in well-controlled bioreactors to perform reproducible fermentations. All batch cultivations of A. oryzae and A. niger were carried out on a defined, minimal medium with glucose or maltose as the sole carbon source. Each species had its own specific 


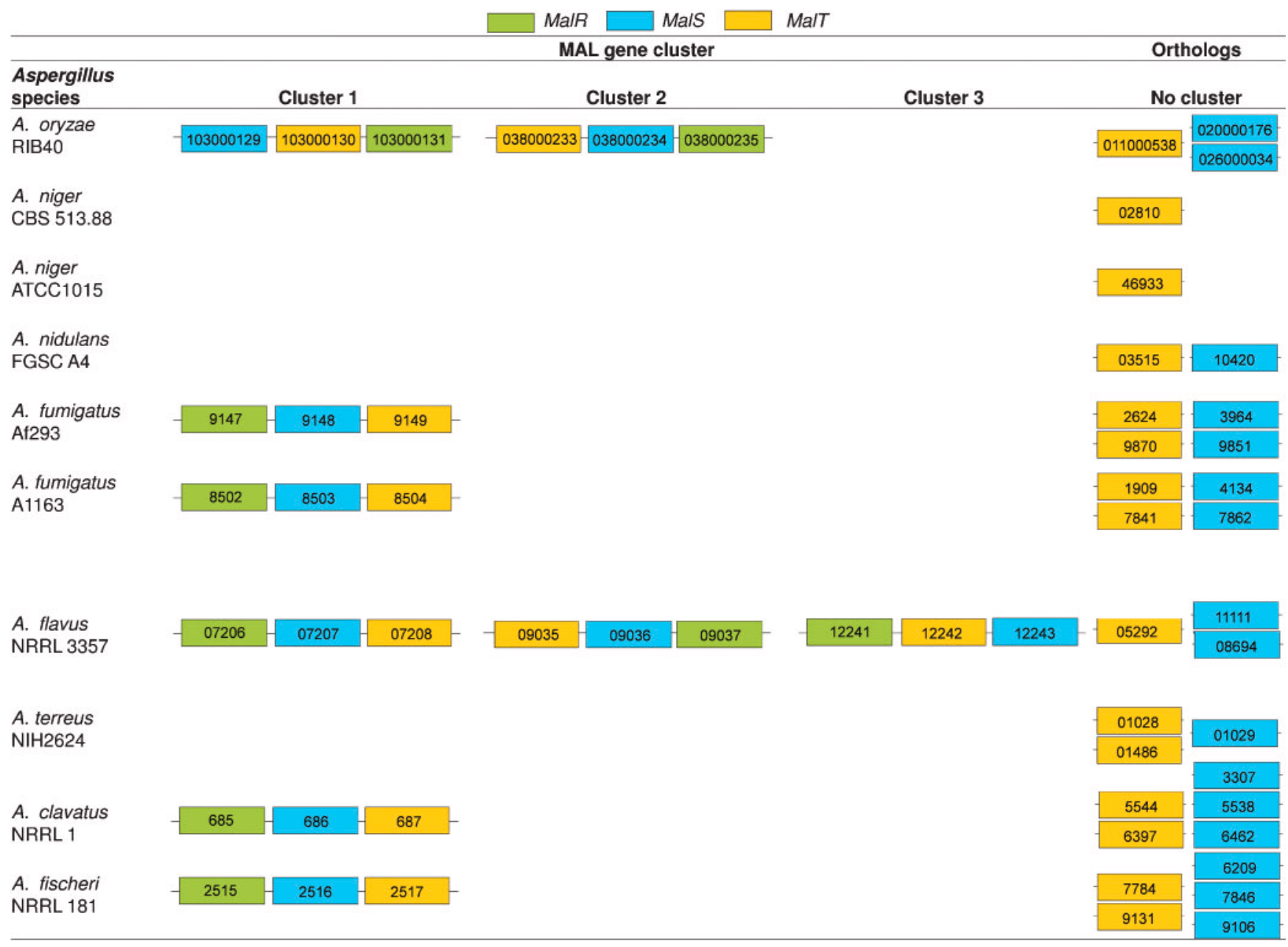

Fig. 1. Diagram showing comparative sequence analysis of the MAL gene cluster between S. cerevisiae and 10 different Aspergillus species. Values in each rectangle represent a shortened ORF name. For the individual full name, the shortened ORF is prefixed by 'AO090' for $A$. oryzae RIB40, 'An02g' for $A$. niger CBS 513.88, 'JGl' for $A$. niger ATCC 1015, 'ANID_' for $A$. nidulans FGSC A4, 'ORF' for A. fumigatus Af293 and A1163, A. clavatus NRRL 1 as well as A. fischeri NRRL 181, 'AFL2T_' for $A$. flavus NRRL 3357, and 'ATET_' for $A$. terreus NIH2624.

cultivation medium optimized for growth of that species (Andersen et al., 2008; Pedersen et al., 2000) (see Methods for details). For each of the two species, three biological replicate cultivations were performed on each carbon source. The results are shown in Fig. 2. Fig. 2(A) shows the biomass growth and substrate concentration profiles of $A$. oryzae and $A$. niger. For both species, these two carbon sources were completely consumed. In comparison to $A$. niger, A. oryzae grew faster than A. niger on both sources, and they were completely consumed at different rates. Glucose was exhausted in $10 \mathrm{~h}$ and maltose in $12 \mathrm{~h}$, at rates of $3.09 \pm 0.02 \mathrm{~g} \mathrm{l}^{-1} \mathrm{~h}^{-1}$ and $2.46 \pm 0.02 \mathrm{~g} \mathrm{l}^{-1} \mathrm{~h}^{-1}$, respectively. The maximum specific growth rate of $A$. oryzae on glucose was $0.38 \pm 0.01 \mathrm{~h}^{-1}$, which is due to the high efficiency in the uptake and metabolism of this sugar. Slightly slower growth was achieved on maltose, where the maximum specific growth rate was $0.32 \pm 0.05 \mathrm{~h}^{-1}$. As shown in Fig. 2, almost no accumulation of glucose was seen in the media during growth on maltose. With A. niger, glucose was exhausted in $32 \mathrm{~h}$ and maltose was consumed after $19 \mathrm{~h}$. As indicated in Fig. 2, growth of A. niger on maltose was faster than that on glucose, where a maximum specific growth rate of $0.22 \pm 0.01 \mathrm{~h}^{-1}$ was achieved. In the case of maltose consumption, there was an accumulation of glucose due to a very high extracellular glucosidase activity expressed by $A$. niger, which allowed the fungus to grow very fast on this carbon source, at a maximum specific growth rate of $0.31 \pm 0.02 \mathrm{~h}^{-1}$. As well as growth rates and biomass yields of $A$. oryzae and A. niger, transcriptional analysis (TA) sampling times and biomass yields at the specific TA sampling time were recorded for the two fungi on the two carbon sources (see Fig. 2B). 
(A)

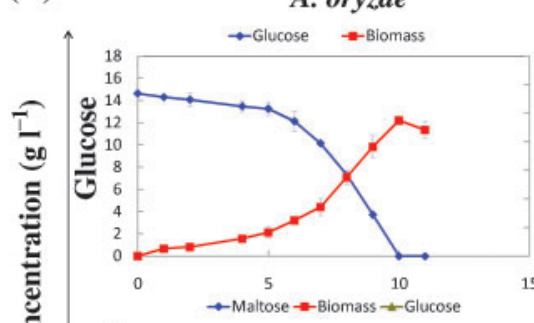

A. oryzae
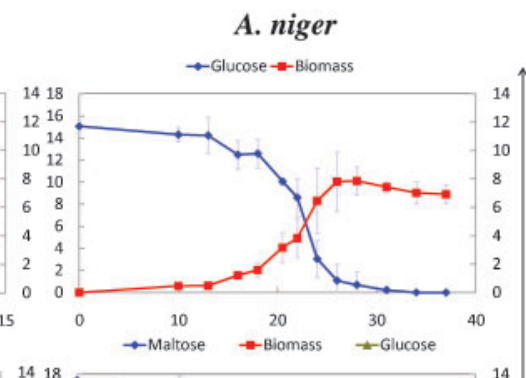
$\rightarrow$ Maltose $\rightarrow$-Biomass $\pitchfork$ Glucose

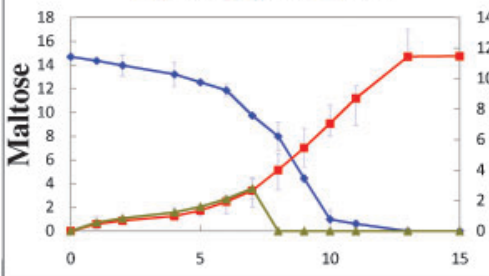

Fermentation time (h)

(B)

\begin{tabular}{llllll}
\hline Strain & $\begin{array}{l}\text { Carbon } \\
\text { source }\end{array}$ & $\begin{array}{l}\mu_{\max } \\
\left(\mathbf{h}^{-1}\right)\end{array}$ & $\begin{array}{l}\boldsymbol{Y}_{\text {sx }} \\
{[\mathrm{g} \mathrm{DW}(\mathrm{g} \mathrm{C}} \\
\left.\text { source })^{-1}\right]\end{array}$ & $\begin{array}{l}\text { Sampling } \\
\text { time for TA } \\
(\mathbf{h})\end{array}$ & $\begin{array}{l}\text { Biomass } \\
\text { for TA } \\
\left(\mathrm{g} \mathrm{DW} \mathrm{kg}^{-1}\right)\end{array}$ \\
\hline A. oryzae & Glucose & $0.38 \pm 0.01$ & $0.51 \pm 0.01$ & $6 \pm 0$ & $2.50 \pm 0.09$ \\
& Maltose & $0.32 \pm 0.05$ & $0.49 \pm 0.05^{*}$ & $7 \pm 0$ & $2.27 \pm 0.09$ \\
A. niger & Glucose & $0.22 \pm 0.01$ & $0.57 \pm 0.05$ & $21 \pm 1$ & $3.74 \pm 0.06$ \\
& Maltose & $0.31 \pm 0.02$ & $0.62 \pm 0.02^{*}$ & $24 \pm 0$ & $3.55 \pm 0.51$ \\
\hline
\end{tabular}

Fig. 2. Biomass and substrate concentration profiles with the different carbon sources (glucose and maltose). (A) Batch cultivation with $A$. oryzae and A. niger. (B) Physiological data for all cultivations, maximum specific growth rate, biomass yield, sampling time for transcriptional analysis (TA), and biomass concentration at the time of sampling for TA are given. Means and standard deviations were calculated from three biological replicates. *Biomass yield was calculated based on glucose [g DW (g glucose) $)^{-1}$.

\section{Comparative transcriptome analysis}

To further test our assumption obtained from comparative genomics for the presence of the MAL regulon existing in A. oryzae and its absence in A. niger, the genome-wide gene expression data obtained from glucose or maltose cultivations were pair-wise compared for each species. To detect transcriptional changes in response to a change in the carbon source, Student's $t$-test statistics were used to identify significantly different gene expression levels with a $P$-value cut-off of 0.05 . This cut-off $P$-value was adjusted by the Benjamini-Hochberg method (Benjamini \& Hochberg, 1995) for correction of multiple testing. Table 1 shows a list of genes that were significantly differentially expressed in A. oryzae between glucose and maltose (16 gene expression changes). In contrast, for $A$. niger, no genes were statistically differentially expressed when using glucose or maltose as carbon source.

\section{Analysis of the MAL regulon}

As shown in Table 1, 16 genes showed higher expression level on maltose compared to glucose in A. oryzae. It is suggested that these genes are induced during metabolism of maltose. Among these genes, 10 protein-encoding genes were functionally annotated and involved in polysaccharide and disaccharide metabolism, such as glucoamylase, maltose permease, maltase, sugar transporters and maltose O-acetyltransferase (EC 2.3.1.79, which acetylates oligosaccharides). In the yeast $S$. cerevisiae, regulation of maltose utilization occurs by the MAL regulon (Chow et al., 1989) via the transcription activator (MALR), which induces maltose permease (MALT) and maltase (MALS) gene expression. Interestingly, among the 16 genes with significantly higher expression on maltose, we found genes encoding maltase in A. oryzae (AO090103000129 and AO090038000234), which correspond to orthologous genes of MALS in S. cerevisiae (see Fig. 1). Moreover, we also found upregulated genes encoding maltose permease, $\mathrm{AO} 090103000130$ and AO090038000233, which are the functionally related orthologous genes of MALT in $S$. cerevisiae (see Fig. 1). The two A. oryzae genes orthologous to the $S$. cerevisiae MALR transcription factor, AO090103000131 and AO090038000235, were also upregulated, but not to a statistically significant extent. This suggests that the mechanism behind the $M A L$ regulon in $A$. oryzae is similar to MALR function in S. cerevisiae, where it is activated by maltose and repressed by glucose. Based on Regulatory Sequence Analysis Tools (RSAT) (ThomasChollier et al., 2008) for identification of the transcription factor binding site in the upstream region of MALR in A. oryzae, we could putatively identify the CreA-binding site 5'-GCGGGG-3' (Cubero \& Scazzocchio, 1994; Drysdale 
Table 1. Pair-wise carbon source comparison for gene expression analysis in $A$. oryzae under the cut-off $P$-value $<0.05$

\begin{tabular}{|llcc|}
\hline Gene name & \multicolumn{1}{c|}{ Protein function } & Fold change & Adjusted $\boldsymbol{P}_{\text {-value }}$ \\
\hline AO090103000130 & Maltose permease & 30.9 & $2.99 \mathrm{E}-06$ \\
AO090103000129 & Maltase & 304.4 & $2.03 \mathrm{E}-05$ \\
AO090701000246 & Hypothetical protein & 9.4 & $1.87 \mathrm{E}-04$ \\
AO090038000233 & Maltose permease & 61.4 & $2.82 \mathrm{E}-04$ \\
AO090038000234 & Maltase & 213.8 & $2.82 \mathrm{E}-04$ \\
AO090012000888 & Maltose O-acetyltransferase & 12.6 & $1.24 \mathrm{E}-03$ \\
AO090003000576 & Hypothetical protein & 6.7 & $5.83 \mathrm{E}-03$ \\
AO090003000321 & Glucoamylase & 9.3 & $7.32 \mathrm{E}-03$ \\
AO090003000256 & Hypothetical protein & 3.1 & $7.32 \mathrm{E}-03$ \\
AO090038000471 & Maltase & 11.7 & $2.92 \mathrm{E}-02$ \\
AO090012000893 & Hypothetical protein & 2.0 & $3.46 \mathrm{E}-02$ \\
AO090011000612 & NAD-binding Rossmann fold oxidoreductase & 1.9 & $3.46 \mathrm{E}-02$ \\
AO090023000245 & Glucose transporter & 9.1 & $3.71 \mathrm{E}-02$ \\
AO090026000775 & Monosaccharide transporter & 4.2 & $3.72 \mathrm{E}-02$ \\
AO090010000746 & Glucoamylase & 2.5 & $3.90 \mathrm{E}-02$ \\
AO090012000896 & Hypothetical protein & 2.0 & $4.39 \mathrm{E}-02$ \\
\hline
\end{tabular}

*All 16 genes showed increased expression on glucose versus maltose.

et al., 1993) in upstream sequences, where this site was found at base position 64 with respect to the start codon of AO090038000235. This result suggests that the presence of glucose represses MALR expression and the formation of the active conformation of the MalR protein as occurs in $S$. cerevisiae (Klein et al., 1996).

According to the results of protein sequence analysis and synteny gene analysis (Sinha \& Meller, 2007) of the MAL gene cluster, we could conclude that $A$. oryzae has two $M A L$ regulons and each regulon contains two genes (i.e. AO090103000131 and AO090038000235) that are likely to be MALR transcriptional activators (see Fig. 1). From this significant evidence combined with the physiological response of $A$. oryzae growth on maltose (see Fig. 2), where the fungus continuously consumed maltose having almost no accumulated glucose over time, we propose that A. oryzae has systematic regulation of maltose utilization by these two MAL regulons, where the MALR transcription factor induces maltose permeases $(M A L T)$ to transport extracellular maltose into the cell and MALR also induces maltase (MALS), which hydrolyses intracellular maltose into glucose, which is then channelled through glycolysis. Fig. 3(A) illustrates the proposed mechanisms for regulation of maltose utilization in A. oryzae.

In contrast, we could not identify any $M A L$ gene cluster in A. niger that is closely homologous to the one in $S$. cerevisiae (see Fig. 1). Furthermore, transcription data analysis of the pair-wise comparison between maltose and glucose in $A$. niger did not show any significant gene expression changes indicating the presence of a $M A L$ cluster. We therefore propose that maltose utilization in $A$. niger most likely does not involve a MAL regulon, but occurs through another regulatory system via the AmyR regulator; the recent publication of genome-wide expression analysis in A. niger by Yuan et al. (2008) supports these results. Fig. 3(B) illustrates the proposed mechanisms for regulation of maltose utilization in $A$. niger.

\section{Key metabolite identification and metabolic subnetwork analysis}

In order to analyse the overall metabolic responses to changes in the carbon source, i.e. using glucose or maltose, we applied the reporter metabolites algorithm to identify key metabolites and to search for highly correlated metabolic subnetworks for the pair-wise comparison (Patil \& Nielsen, 2005). This analysis relied on the reconstructed genomescale metabolic network of A. oryzae (Vongsangnak et al., 2008), and therefore we demonstrated how these metabolic networks can be used to map regulatory responses in this Aspergillus sp. The top 15 high-scoring key metabolites for A. oryzae are listed in Table 2. To identify high-scoring metabolic subnetworks, we performed subnetwork analysis using the whole reaction set from the reconstructed metabolic network of $A$. oryzae. Fig. 4 shows the list of key genes encoding enzymes and transporters comprising the subnetwork of $A$. oryzae investigated upon a change of carbon source, from glucose to maltose.

\section{DISCUSSION}

Maltose is of economic relevance due to the fact that it is one of the products of starch hydrolysis and it is used as an inducer for protein production in Aspergillus species. In this work, we conducted comparative genomics and transcriptomics in $A$. oryzae or $A$. niger in order to 

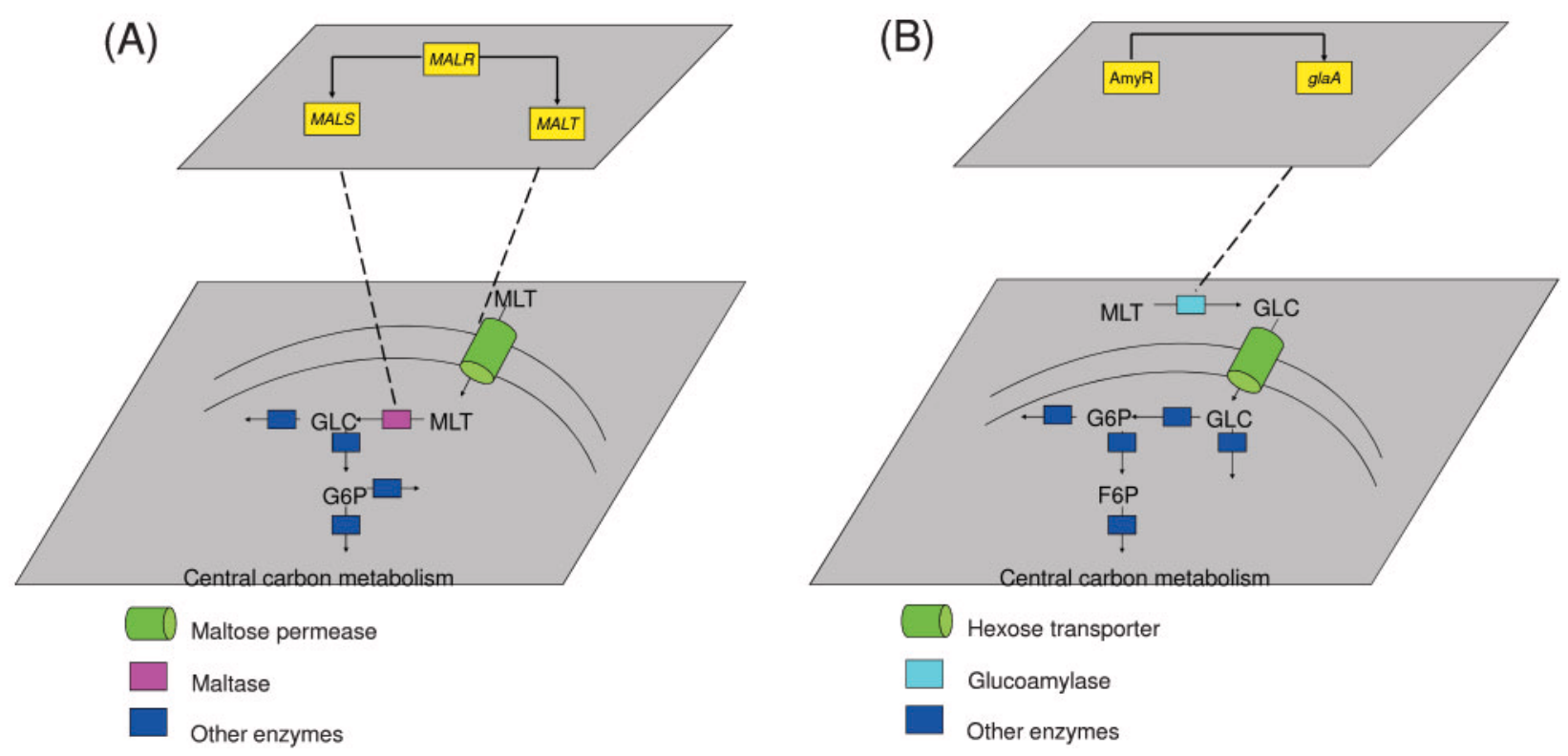

Fig. 3. Diagram showing maltose utilization and regulation in $A$. oryzae (A) and A. niger (B).

investigate maltose uptake, metabolism and regulation. In $S$. cerevisiae, maltose utilization and regulation occurs through the use of the MAL regulon. This regulon is composed of three genes, encoding maltase $(M A L S)$, a maltose transmembrane transporter (MALT), and a positive regulator (MALR) (Novak et al., 2004). Interestingly, some bacteria such as Lactococcus lactis (Andersson \& Rådström, 2002) and Streptomyces coelicolor (van Wezel et al., 1997) also make use of the MAL

Table 2. List of significant key metabolites from the pair-wise carbon source comparison (maltose vs glucose) in $A$. oryzae $(P$-value cut-off $<0.01)$

\begin{tabular}{|lc|}
\hline Key metabolite & $\boldsymbol{P}$-value \\
\hline Maltose & $0.00 \mathrm{E}+00$ \\
Maltose (extracellular) & $0.00 \mathrm{E}+00$ \\
Maltotriose & $3.04 \mathrm{E}-14$ \\
Glucose & $1.18 \mathrm{E}-11$ \\
Glucose (extracellular) & $1.44 \mathrm{E}-09$ \\
Glycogen (extracellular) & $2.50 \mathrm{E}-06$ \\
Starch (extracellular) & $2.50 \mathrm{E}-06$ \\
D-Fructose (extracellular) & $3.73 \mathrm{E}-05$ \\
2-Keto-myo-inositol & $4.22 \mathrm{E}-05$ \\
D-Galactose & $7.41 \mathrm{E}-05$ \\
D-Aspartate & $1.16 \mathrm{E}-04$ \\
D-Arabinono-1,4-lactone & $1.60 \mathrm{E}-04$ \\
$\mathrm{H}_{2} \mathrm{O}$ (extracellular) & $4.88 \mathrm{E}-04$ \\
D-Mannose (extracellular) & $5.85 \mathrm{E}-04$ \\
$\alpha, \alpha$-Trehalose (extracellular) & $9.11 \mathrm{E}-04$ \\
\hline
\end{tabular}

regulatory system involving the MALR transcriptional activator of their corresponding operons for maltose utilization. Through comparative genomics analysis we were able to identify two MAL clusters in A. oryzae, but no $M A L$ cluster in A. niger. We also found one MAL cluster in A. clavatus, A. fumigatus and A. fischeri as well as three $M A L$ clusters in A. flavus.

We further confirmed the presence of $M A L$ clusters in $A$. oryzae at the transcriptional level. The results from genome-wide expression analysis identified a subset of 16 genes to be significantly upregulated during growth on maltose. Among them, we found MALS encoding maltase and MALT encoding maltose permease. In addition, according to gene expression data, we also found higher expression levels of MALR encoding a transcriptional activator in maltose utilization, but the expression changes were not statistically significant. Based on our integrated data analysis, we could conclude that $A$. oryzae can utilize maltose via the $M A L$ regulon system.

We further investigated the transcriptional responses to change in the carbon source in batch cultivations with glucose or maltose through the use of reporter metabolites and subnetwork analysis (Patil \& Nielsen, 2005) together with the reconstructed metabolic network of A. oryzae (Vongsangnak et al., 2008). The transcriptional responses were in general consistent with the changes expected at the phenotypic level, indicating that regulation at the transcriptional level plays a significant role in the overall regulation during growth on these two carbon sources.

In comparison to A. oryzae, comparative genome analysis showed that $A$. niger does not have a MAL cluster. We 


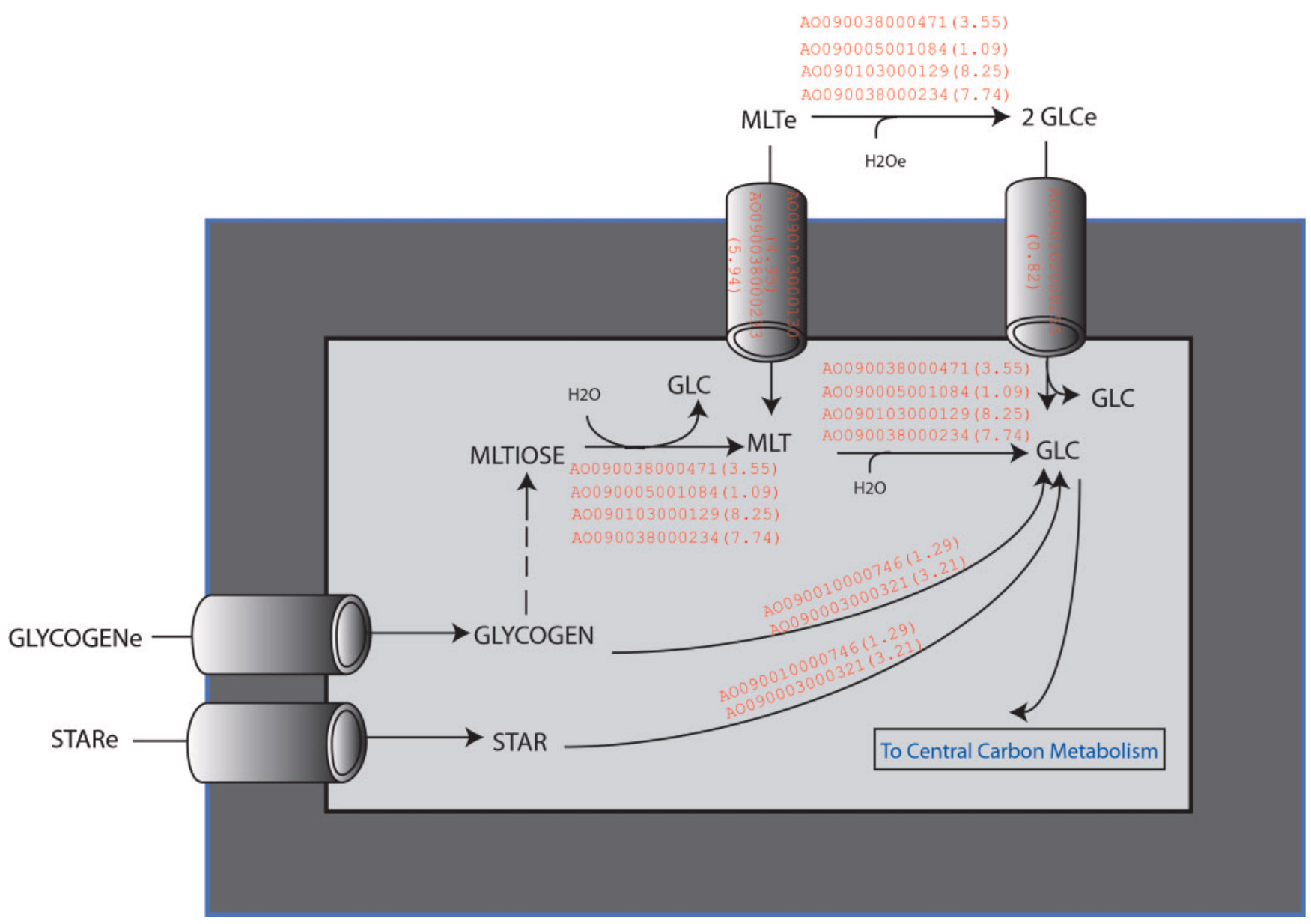

Fig. 4. Small metabolic subnetwork from pair-wise carbon source comparison in A. oryzae: key metabolites, key enzymes and transporters in the small subnetwork identified for a change of carbon source, from glucose to maltose. Gene names in red are upregulated upon the change; numbers in parentheses are $\log _{2}$ fold-change value upon the change of carbon source. The abbreviations of metabolites are as follows: GLC, glucose; MLT, maltose; MLTIOSE, maltotriose; STAR, starch; GLYCOGEN, glycogen; $\mathrm{H} 2 \mathrm{O}$, water. Extracellular metabolites are followed by 'e'.

further evaluated the expression level of the MAL gene cluster by transcriptome analysis. These results also showed absence of a $M A L$ gene cluster in A. niger. Our results are consistent with previous transcriptome studies in A. niger using the wild-type strain N402 (ATCC 9029) (Yuan et al., 2008), which did not identify upregulation of components of the $M A L$ regulon when comparing gene expression data on xylose versus maltose cultivations. Moreover, we did not find any significant changes in gene expression in $A$. niger when comparing maltose to glucose, although $A$. niger glaA is proposed to be strongly induced on maltose, as described earlier by Fowler et al. (1990). In order to utilize maltose, we propose that $A$. niger makes use of another regulatory system via the AmyR regulator. Yuan et al. (2008) suggested that AmyR is an important transcription factor that is found in A. niger and that $a m y R$ itself is induced by the presence of maltose. In addition, their studies indicated that regulation of $a m y R$ transcription takes place in $A$. niger and showed that a disruption of the AmyR transcription factor resulted in low levels of extracellular enzymes, i.e. acid $\alpha$-amylase (AamA), $\alpha$-glucosidases (AgdA and a putative $\mathrm{AgdB}$ ) and glucoamylase (GlaA), converting maltose to glucose and consequently activating a stress response due to low glucose levels. The low availability of glucose transferred a signal to downregulate glucose transporters (Yuan et al., 2008). This is in agreement with our transcriptome results and the physiological response obtained in $A$. niger maltose cultivations (see Fig. 2), where maltose cleavage occurred faster than glucose uptake and metabolism, leading to a high extracellular accumulation of glucose over time. Our findings therefore support the conclusions from previous studies that $A$. niger regulates maltose utilization by the AmyR transcriptional activator. AmyR activates genes encoding known extracellular starch-degrading enzymes, such as aamA, glaA, agdA and a putative $\alpha$-glucosidaseencoding gene, agdB. The glaA gene product, an extracellular glucoamylase, can convert extracellular maltose to 
extracellular glucose and then glucose can be taken up by glucose transporters.

Interestingly, maltose has been found to be better than glucose for glucoamylase production, but it has been reported as well that this is a strain-dependent phenomenon: for some strains there is no difference between the use of glucose and maltose (Schrickx et al., 1993). Furthermore, studies of glaA regulation in A. niger strain ATCC 10864 showed starch, maltose and glucose as positive inducers of glucoamylase production (Fowler et al., 1990). In contrast, previous studies with the A. niger BO1 strain used in this study have reported no difference between maltose and glucose as carbon source with respect to glucoamylase productivity as well as identical mRNA levels for growth on maltose or glucose in chemostat cultivations (Pedersen et al., 2000). Based on findings in the literature (Yuan et al., 2008) and our findings, we suggest that $A$. niger utilizes maltose by means of extracellular hydrolysis by glucoside hydrolases such as glucoamylase followed by glucose uptake and metabolism.

From comparative genome and transcriptome analysis, we showed that maltose utilization and regulation of $A$. oryzae is very similar to that found in the yeast $S$. cerevisiae for sugar degradation pathways where a number of enzymes and proteins are involved. Our analysis should help in understanding how maltose is utilized and regulated in Aspergillus species and to convey improvements in industrial practice for protein production in the future.

\section{ACKNOWLEDGEMENTS}

The authors would like to thank Lone Vuholm and Anne Kejser Jensen at Novozymes as well as Tina Johansen, Pia Friis and Lene Christiansen at the Technical University of Denmark for technical assistance. Finally, we would like to thank Novozymes Bioprocess Academy and the Chalmers University of Technology Foundation for financial support to W.V. and the National Council of Research Conacyt-Mexico and Chalmers University of Technology Foundation for financial support to M.S.

\section{REFERENCES}

Affymetrix \& GeneChip (2007). Affymetrix Genechip Expression Analysis Technical Manual. P/N 702232, Affymetrix, Santa Clara, CA, Revision 2.

Altschul, S. F., Gish, W., Miller, W., Myers, E. W. \& Lipman, D. J. (1990). Basic local alignment search tool. J Mol Biol 215, 403-410.

Andersen, M. R., Vongsangnak, W., Panagiotou, G., Margarita, P. S., Lehmann, L. \& Nielsen, J. (2008). A tri-species Aspergillus microarray - advancing comparative transcriptomics. Proc Natl Acad Sci U S A 105, 4387-4392.

Andersson, U. \& Rådström, P. (2002). Physiological function of the maltose operon regulator, MalR, in Lactococcus lactis. BMC Microbiol 2, 28.

Baker, S. E. (2006). Aspergillus niger genomics: past, present and into the future. Med Mycol 44, S17-S21.

Baker, S. E. \& Bennett, J. (2008). An overview of the genus Aspergillus. In The Aspergilli: Genomics, Medical Aspects, Biotechnology, and Research Methods, pp. 3-13. Edited by S. A. Osmani \& G. H. Goldman. Boca Raton, FL: CRC Press.

Barton, L. L., Georgi, C. E. \& Lineback, D. R. (1972). Effect of maltose on glucoamylase formation by Aspergillus niger. J Bacteriol 111, 771777.

Benjamini, Y. \& Hochberg, Y. (1995). Controlling the false discovery rate - a practical and powerful approach to multiple testing. $J R$ Statist Soc B 57, 289-300.

Breakspear, A. \& Momany, M. (2007). The first fifty microarray studies in filamentous fungi. Microbiology 153, 7-15.

Carlsen, M. \& Nielsen, J. (2001). Influence of carbon source on alphaamylase production by Aspergillus oryzae. Appl Microbiol Biotechnol 57, 346-349.

Carlsen, M., Nielsen, J. \& Villadsen, J. (1996). Growth and alphaamylase production by Aspergillus oryzae during continuous cultivations. J Biotechnol 45, 81-93.

Chow, T. H. C., Sollitti, P. \& Marmur, J. (1989). Structure of the multigene family of Mal loci in Saccharomyces. Mol Gen Genet 217, 60-69.

Cubero, B. \& Scazzocchio, C. (1994). Two different, adjacent and divergent zinc finger binding sites are necessary for CreA mediated carbon catabolite repression in the proline gene cluster of Aspergillus nidulans. EMBO J 13, 407-415.

Drysdale, M. R., Kolze, S. E. \& Kelly, J. M. (1993). The Aspergillus niger carbon catabolite repressor gene, creA. Gene 130, 241-245.

Dudoit, S., Gendeman, R. C. \& Quackenbush, J. (2003). Open source software for the analysis of microarray data. Biotechniques (Suppl.), 45-51.

Fedorova, N. D., Khaldi, N., Joardar, V. S., Maiti, R., Amedeo, P., Anderson, M. J., Crabtree, J., Silva, J. C., Badger, J. H. \& other authors (2008). Genomic islands in the pathogenic filamentous fungus Aspergillus fumigatus. PLoS Genet 4, e1000046.

Fowler, T., Berka, R. M. \& Ward, M. (1990). Regulation of the glaA gene of Aspergillus niger. Curr Genet 18, 537-545.

Galagan, J. E., Calvo, S. E., Cuomo, C., Ma, L. J., Wortman, J. R., Batzoglou, S., Lee, S. I., Baştürkmen, M., Spevak, C. C. \& other authors (2005). Sequencing of Aspergillus nidulans and comparative analysis with A. fumigatus and A. oryzae. Nature 438, 1105-1115.

Gautier, L., Cope, L., Bolstad, B. M. \& Irizarry, R. A. (2004). affy analysis of Affymetrix GeneChip data at the probe level. Bioinformatics 20, 307-315.

Gentleman, R. C., Carey, V. J., Bates, D. M., Bolstad, B., Dettling, M., Dudoit, S., Ellis, B., Gautier, L., Ge, Y. \& other authors (2004). Bioconductor: open software development for computational biology and bioinformatics. Genome Biol 5, R80.

Irizarry, R. A., Hobbs, B., Collin, F., Beazer-Barclay, Y. D., Antonellis, K. J., Scherf, U. \& Speed, T. P. (2003). Exploration, normalization, and summaries of high density oligonucleotide array probe level data. Biostatistics 4, 249-264.

Kato, N., Murakoshi, Y., Kato, M., Kobayashi, T. \& Tsukagoshi, N. (2002). Isomaltose formed by alpha-glucosidases triggers amylase induction in Aspergillus nidulans. Curr Genet 42, 43-50.

Klein, C. J. L., Olsson, L., Ronnow, B., Mikkelsen, J. D. \& Nielsen, J. (1996). Alleviation of glucose repression of maltose metabolism by MIG1 disruption in Saccharomyces cerevisiae. Appl Environ Microbiol 62, 4441-4449.

Machida, M., Asai, K., Sano, M., Tanaka, T., Kumagai, T., Terai, G., Kusumoto, K., Arima, T., Akita, O. \& other authors (2005). Genome sequencing and analysis of Aspergillus oryzae. Nature 438, 1157-1161.

Magnuson, J. K. \& Lasure, L. L. (2004). Organic acid production by filamentous fungi. In Advances in Fungal Biotechnology for Industry, 
Agriculture, and Medicine, pp. 307-340. Edited by J. S. Tkacz \& L. Lange. New York: Kluwer Academic/Plenum Publishers.

Needleman, R. B., Kaback, D. B., Dubin, R. A., Perkins, E. L., Rosenberg, N. G., Sutherland, K. A., Forrest, D. B. \& Michels, C. A. (1984). MAL6 of Saccharomyces: a complex genetic locus containing three genes required for maltose fermentation. Proc Natl Acad Sci U S A 81, 2811-2815.

Nierman, W. C., Pain, A., Anderson, M. J., Wortman, J. R., Kim, H. S., Arroyo, J., Berriman, M., Abe, K., Archer, D. B. \& other authors (2005). Genomic sequence of the pathogenic and allergenic filamentous fungus Aspergillus fumigatus. Nature 438, 1151-1156.

Novak, S., Zechner-Krpan, V. \& Maric, V. (2004). Regulation of maltose transport and metabolism in Saccharomyces cerevisiae. Food Technol Biotechnol 42, 213-218.

Patil, K. R. \& Nielsen, J. (2005). Uncovering transcriptional regulation of metabolism by using metabolic network topology. Proc Natl Acad Sci U S A 102, 2685-2689.

Payne, G. A., Nierman, W. C., Wortman, J. R., Pritchard, B., Brown, D., Dean, R., Bhatnagar, D., Cleveland, T., Machida, M. \& Yu, J. (2006). Whole genome comparison of Aspergillus flavus and A. oryzae. Med Mycol 44, S9-S11.

Pedersen, H., Beyer, M. \& Nielsen, J. (2000). Glucoamylase production in batch, chemostat and fed-batch cultivations by an industrial strain of Aspergillus niger. Appl Microbiol Biotechnol 53, 272-277.

Pel, H. J., de Winde, J. H., Archer, D. B., Dyer, P. S., Hofmann, G., Schaap, P. J., Turner, G., de Vries, R. P., Albang, R. \& other authors (2007). Genome sequencing and analysis of the versatile cell factory Aspergillus niger CBS 513.88. Nat Biotechnol 25, 221-231.

Schrickx, J. M., Krave, A. S., Verdoes, J. C., Vandenhondel, C., Stouthamer, A. H. \& Vanverseveld, H. W. (1993). Growth and product formation in chemostat and recycling cultures by Aspergillus niger N402 and a glucoamylase overproducing transformant, provided with multiple copies of the glaA gene. J Gen Microbiol 139, 2801-2810.
Sinha, A. U. \& Meller, J. (2007). Cinteny: flexible analysis and visualization of synteny and genome rearrangements in multiple organisms. BMC Bioinformatics $\mathbf{8}, 82$.

Smyth, G. K., Michaud, J. \& Scott, H. S. (2005). Use of within-array replicate spots for assessing differential expression in microarray experiments. Bioinformatics 21, 2067-2075.

Thomas-Chollier, M., Sand, O., Turatsinze, J. V., Janky, R., Defrance, M., Vervisch, E., Brohee, S. \& van Helden, J. (2008). RSAT: regulatory sequence analysis tools. Nucleic Acids Res 36, W119-W127.

van Wezel, G. P., White, J., Young, P., Postma, P. W. \& Bibb, M. J. (1997). Substrate induction and glucose repression of maltose utilization by Streptomyces coelicolor A3(2) is controlled by malR, a member of the lacl-galR family of regulatory genes. Mol Microbiol 23, 537-549.

Vongsangnak, W., Olsen, P., Hansen, H., Krogsgaard, S. \& Nielsen, J. (2008). Improved annotation through genome-scale metabolic modeling of Aspergillus oryzae. BMC Genomics 9, 245.

Workman, C., Jensen, L. J., Jarmer, H., Berka, R., Gautier, L., Nielser, H. B., Saxild, H. H., Nielsen, C., Brunak, S. \& Knudsen, S. (2002). A new non-linear normalization method for reducing variability in DNA microarray experiments. Genome Biol 3, research0048.

Wortman, J. R., Gilsenan, J. M., Joardar, V., Deegan, J., Clutterbuck, J., Andersen, M. R., Archer, D., Bencina, M., Braus, G. \& other authors (2009). The 2008 update of the Aspergillus nidulans genome annotation: a community effort. Fungal Genet Biol 46, S2-S13.

Yu, J., Cleveland, T., Nierman, W. \& Bennett, J. (2005). Aspergillus flavus genomics: gateway to human and animal health, food safety, and crop resistance to diseases. Rev Iberoam Micol 22, 194-202.

Yuan, X. L., van der Kaaij, R. M., van den Hondel, C., Punt, P. J., van der Maarel, M., Dijkhuizen, L. \& Ram, A. F. J. (2008). Aspergillus niger genome-wide analysis reveals a large number of novel alpha-glucan acting enzymes with unexpected expression profiles. Mol Genet Genomics 279, 545-561.

Edited by: B. A. Horwitz 